\title{
Silicon Photomultipliers for the decay tunnel instrumentation of the ENUBET neutrino beam
}

\author{
M. Pozzato i,*, F. Acerbi a, G. Ballerini i,c, A. Berra ${ }^{\text {b,c }}$, M. Bonesini c , A. Branca ${ }^{\text {e,j }}$, C. Brizzolari ${ }^{\text {c,d }}$, \\ G. Brunetti ${ }^{\text {e }}$, M. Calviani ${ }^{\text {f }}$, S. Capelli ${ }^{\text {b,c }}{ }^{\text {, S. Carturan }}{ }^{\text {g }}$, M.G. Catanesi ${ }^{\text {h }}$, S. Cecchini ${ }^{\text {i }}$, \\ N. Charitonidis ${ }^{\mathrm{f}}$, F. Cindolo ${ }^{\mathrm{i}}$, G. Collazuol ${ }^{\mathrm{e}, \mathrm{j}}$, E. Conti ${ }^{\mathrm{e}}$, F. Dal Corso ${ }^{\mathrm{e}}$, C. Delogu ${ }^{\mathrm{c}, \mathrm{d}}$, G. De Rosa ${ }^{\mathrm{k}}$, \\ A. Falcone ${ }^{c, d}$, A. Gola ${ }^{a}$, C. Jollet ${ }^{1, n}$, V. Kain ${ }^{f}$, B. Klicek ${ }^{\mathrm{t}}$, Y. Kudenko ${ }^{\mathrm{m}}$, M. Laveder ${ }^{\mathrm{e}, \mathrm{j}}$, \\ A. Longhin ${ }^{e, j}$, L. Ludovici ${ }^{\circ}$, E. Lutsenko ${ }^{b, c}$, L. Magaletti $^{\text {h }}$, G. Mandrioli $^{\mathrm{i}}$, A. Margotti ${ }^{\mathrm{i}}$, \\ V. Mascagna ${ }^{\text {b,c }}$, N. Mauri ${ }^{\mathrm{i}, \mathrm{r}}$, L. Meazza ${ }^{\mathrm{c}, \mathrm{d}}$, A. Meregaglia ${ }^{\mathrm{n}, \mathrm{p}}$, M. Mezzetto ${ }^{\mathrm{e}}$, M. Nessi ${ }^{\mathrm{f}}$, A. Paoloni ${ }^{\mathrm{q}}$, \\ M. Pari e,j, E.G. Parozzi ${ }^{c, d}$, L. Pasqualini i, ${ }^{\mathrm{i}, \mathrm{A}}$, G. Paternoster ${ }^{\mathrm{a}}$, L. Patrizii ${ }^{\mathrm{i}}$, M. Prest ${ }^{\mathrm{b}, \mathrm{c}}$, F. Pupilli $^{\mathrm{e}}$, \\ E. Radicioni ${ }^{\text {h }}$, C. Riccio ${ }^{\text {k,s }}$, A.C. Ruggieri ${ }^{k}$, C. Scian ${ }^{j}$, G. Sirri ${ }^{i}$, M. Soldani ${ }^{\text {b,c }}$, M. Stipcevic ${ }^{t}$, \\ M. Tenti ${ }^{i}$, F. Terranova ${ }^{c, d}$, M. Torti ${ }^{c, d}$, E. Vallazza ${ }^{c}$, F. Velotti ${ }^{f}$, M. Vesco ${ }^{g}$, L. Votano $^{q}$
}

a Fondazione Bruno Kessler (FBK) and INFN TIFPA, Trento, Italy

${ }^{\mathrm{b}}$ Phys. Dep., Università degli studi dell'Insubria, via Valeggio 11, Como, Italy

c INFN, Sezione di Milano-Bicocca, piazza della Scienza 3, Milano, Italy

d Phys. Dep. Università di Milano-Bicocca, piazza della Scienza 3, Milano, Italy

e INFN Sezione di Padova, via Marzolo 8, Padova, Italy

${ }^{\mathrm{f}}$ CERN, 1211 Geneva 23, Switzerland

${ }^{\mathrm{g}}$ INFN Laboratori Nazionali di Legnaro, Viale dell'Università, 2 - Legnaro (PD), Italy

${ }^{\mathrm{h}}$ INFN Sezione di Bari, via Amendola 173, Bari, Italy

${ }^{\mathrm{i}}$ INFN, Sezione di Bologna, viale Berti-Pichat 6/2, Bologna, Italy

${ }^{\mathrm{j}}$ Phys. Dep. Università di Padova, via Marzolo 8, Padova, Italy

${ }^{\mathrm{k}}$ INFN, Sezione di Napoli, via Cinthia, 80126, Napoli, Italy

${ }^{1}$ IPHC, Université de Strasbourg, CNRS/IN2P3, Strasbourg, France

${ }^{\mathrm{m}}$ Institute of Nuclear Research of the Russian Academy of Science, Moscow, Russia

${ }^{\mathrm{n}}$ Centre de Etudes Nucleaires de Bordeaux Gradignan, 19 Chemin du Solarium, Bordeaux, France

${ }^{\circ}$ INFN, Sezione di Roma 1, piazzale A. Moro 2, Rome, Italy

${ }^{\mathrm{P}}$ CENBG, Université de Bordeaux, CNRS/IN2P3, 33175 Gradignan, France

q INFN, Laboratori Nazionali di Frascati, via Fermi 40, Frascati (Rome), Italy

${ }^{\mathrm{r}}$ Phys. Dep. Università di Bologna, viale Berti-Pichat 6/2, Bologna, Italy

${ }^{s}$ Phys. Dep. Università degli Studi di Napoli Federico II, via Cinthia, 80126, Napoli, Italy

${ }^{t}$ Center of Excellence for Advanced Materials and Sensing Devices, Ruder Boskovic Institute, HR-10000 Zagreb, Croatia

\section{A B S T R A C T}

The goal of the ENUBET project is to demonstrate that a precision of $\sim 1 \%$ on measurement of the absolute neutrino cross section at GeV scale can be achieved by monitoring the positron production in the decay tunnel coming from the three-body semileptonic decays of kaons. The baseline option for the tunnel instrumentation employs a fine-grained shashlik calorimeter with a $4.3 \mathrm{X}_{0}$ longitudinal segmentation to separate positrons and pions coming from other decay modes of kaons. The system is complemented by rings of plastic scintillator doublets below the calorimeter acting as a photon veto to suppress the $\pi^{0}$ background and to provide timing informations. SiPMs instrumenting the detector will be exposed to sizeble amounts of neutrons arising in hadronic showers. In order to reproduce such a working environment, SiPMs with different cell size (from 12 to $20 \mu \mathrm{m}$ ) were irradiated at the INFN-LNL CN Van Der Graaf with neutron fluences up to $2 \times 10^{11} \mathrm{n} / \mathrm{cm}^{2}$ (1 MeV-eq.). The exposed light sensors were characterized in terms of I-V curves at different irradiation levels, and their response tested by exposing a prototype on beam at CERN. In this contribution we will report the results of the described tests on SiPMs, together with the advances in their integration with the ENUBET detectors.

\footnotetext{
* Corresponding author.

E-mail address: michele.pozzato@bo.infn.it (M. Pozzato).
} 


\section{Introduction}

Physical motivation. Neutrinos played a fundamental role in the study of weak interactions and opened a window beyond the Standard Model after the discovery of neutrino oscillation. The current neutrino experiments are affected by some intrinsic limitation in cross section measurements due to the challenging task of predicting neutrino flux which rely on a detailed simulation of the beamline elements and on hadronic physics. Even if data driven constraints to the simulation have been employed in the past, the flux prediction preserves a critical link with the simulation leading to systematics $(\mathrm{O}(10 \%))$. Flux uncertainties represent the common limitation for all neutrino cross section precision measurements which will play a key role in the next generation of oscillation physics experiments with a significant impact on the study of $\mathrm{CP}$ violation and mass hierarchy problems. A breakthrough in the field could be achieved by monitoring the production of positron from the $K_{e 3}$ decays directly in the decay tunnel: this new method can provide a pure source of $v_{e}$ by using an observable (the positron rate) which is directly linked to the $v_{e}$ rate at far detector. The direct monitoring of the neutrino rate at the source is expected to be capable to provide a flux measurement error of $\sim 1 \%$ [1].

The ENUBET project. The ENUBET project (Enhanced NeUtrino BEams from kaon Tagging) [2,3], funded by the European Research Council, ${ }^{1}$ is aimed at demonstrating the technical feasibility and physics performance of monitored neutrino beams. This will be done by performing the following tasks:

- design and construct a detector capable of performing positron identification in the harsh environment of a neutrino beam decay tunnel

- studying the precise layout of the kaons and pions focusing and transport system with suitable proton extraction schemes.

\section{Beamline}

The proposed setup consists of primary protons impinging on a target to produce secondary hadrons which are delivered to a secondary instrumented beamline after charge and momentum selection (Fig. 1).

The secondary beam is designed and optimized to have a central momentum of $8.5 \mathrm{GeV}$ and a momentum bite of $10 \%$ to keep low the decaying in the transfer line. Moreover these energies are also high enough to keep the contamination of $v_{e}$ from $\mu$ decays below $2 \%$. The necessity to prevent undecayed secondaries or $\mu$ coming from $\pi$ decay from hitting the tagger causing unmanageable pile-up effects is a guiding factor in tuning the emittance at the entrance of the decay tunnel to few mrad over $10 \times 10 \mathrm{~cm}^{2}$.

\section{Instrumented decay tunnel}

In the baseline design the positron tagger is shaped as a hollow cylinder surrounding a fraction of the decay tunnel and based on a shashlik calorimeter [4]. The instrumentation is complemented with rings of plastic scintillator doublets below the calorimeter acting as a photon veto to suppress the $\pi^{0}$ background and providing timing informations (Fig. 2).

The tagger has a $4.3 X_{0}$ longitudinal segmentation to separate positrons and pions coming from other decay modes of kaons [5]. This is a cost effective solution with a basic unit, named UCM, composed of 5 layers of plastic scintillators $\left(3 \times 3 \times 0.5 \mathrm{~cm}^{3}\right.$ each) interleaved with iron slabs $\left(3 \times 3 \times 1.5 \mathrm{~cm}^{3}\right.$ each) crossed by 9 Wavelength Shifting (WLS) fibers. These fibers are directly coupled to small-area SiPMs hosted on a PCB on the back of each module, avoiding dead zones due to fiber bundling (Fig. 3).

Concerning the photon veto, each tile $\left(3 \times 3 \times 0.5 \mathrm{~cm}^{3}\right)$ is readout by two lateral WLS fibers optically linked to a single SiPM (see Fig. 12).

1 ERC Consolidator Grant,G.A. 681647, P.I. A. Longhin, host institution Padova University and INFN as beneficiary. Since march 2019, ENUBET is also a CERN Neutrino Platform experiment (NP06/ENUBET).

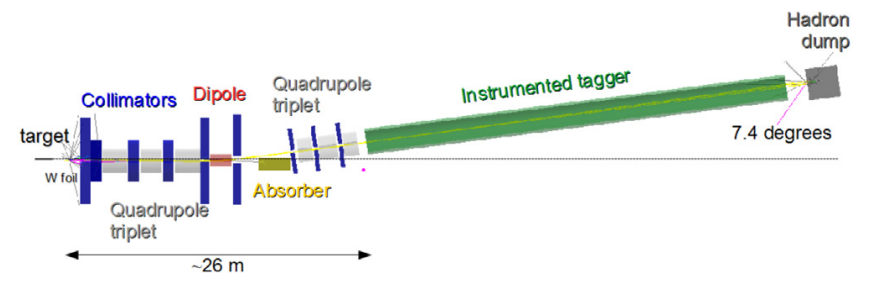

Fig. 1. Beamline layout.
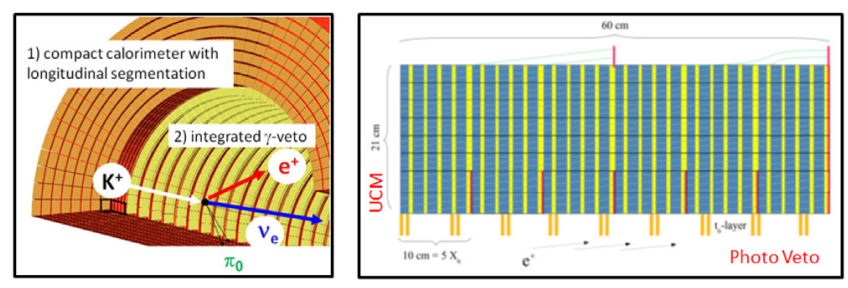

Fig. 2. Left: Sketch of the hollow cylinder tagger geometry. Right: Longitudinal section of the calorimeter plus photon veto modules.
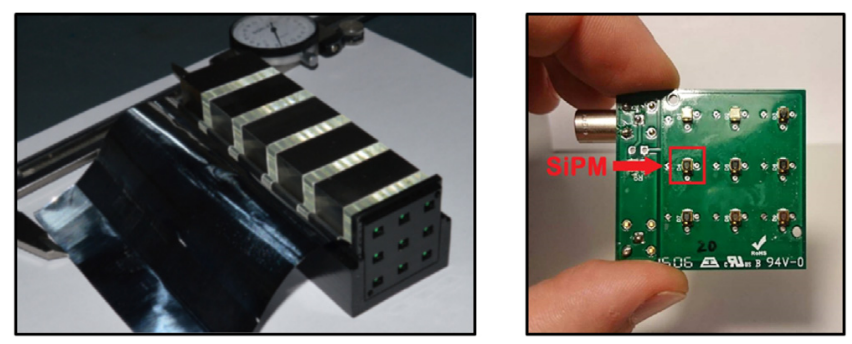

Fig. 3. Left: one of the prototypes of the shashlik calorimeter modules instrumenting the decay tunnel. Right: a PCB with the SiPMs reading the 9 WLS fibers of a UCM.

\section{Irradiation tests}

SiPMs instrumenting the calorimeter will be exposed to a large flux of neutrons coming from the hadronic showers [6]. The integrated fluences to collect a sample of about $10^{4} v_{e}$ CC interactions depend on the position of the calorimeter with respect to the axis of the secondary beam as shown in Fig. 4. At $1 \mathrm{~m}$ distance the non-ionizing fluence integrated during the lifetime of the experiment and scaled to $1 \mathrm{MeV}$ equivalent neutrons is $1.8 \times 10^{11} \mathrm{n} / \mathrm{cm}^{2}$. The ionizing dose is $0.06 \mathrm{kGy}$.

In order to test the functionality of the silicon photomultiplier over the entire lifetime of the experiment, SiPMs with different cell size (from 12 to $20 \mu \mathrm{m}$ ) produced by Fondazione Bruno Kessler (FBK) employing the RGB-HD technology were exposed at the INFN-LNL. This general purpose facility is based on the $\mathrm{CN}$ van der Graaf accelerator: with a maximum voltage of $7 \mathrm{MV}$ it can accelerate protons and other light nuclei with currents up to $5 \mu \mathrm{A}$. For these tests a beam of protons are sent on a thick Beryllium target and produces neutrons through the $\mathrm{Be}(\mathrm{p}, \mathrm{xn})$ reactions; the irradiated sample is located inside an experimental area with an external shield of concrete and an inner shell of water as neutron moderator (Fig. 5 ).

The irradiated sample consisted of three PCB boards used for the ENUBET UCMs, each one hosting 9 SiPMs and integrating passive components and signal routing toward the front-end electronics. The PCB boards host SiPM with 12, 15, $20 \mu \mathrm{m}$ cells respectively. The SiPMs belonging to the same PCB board are connected in parallel and readout without amplification. The temperature of the sample during and after the irradiation was measured with LM35 temperature sensors. In addition, a test PCB with a single $1 \mathrm{~mm}^{2}, 12 \mu \mathrm{m}$ pitch SiPM is used to evaluate the single photoelectron sensitivity by means of an Advansid trans-impedance amplifier (ASD-EP-EB-N) and a Rohde \& Schwarz RTO 


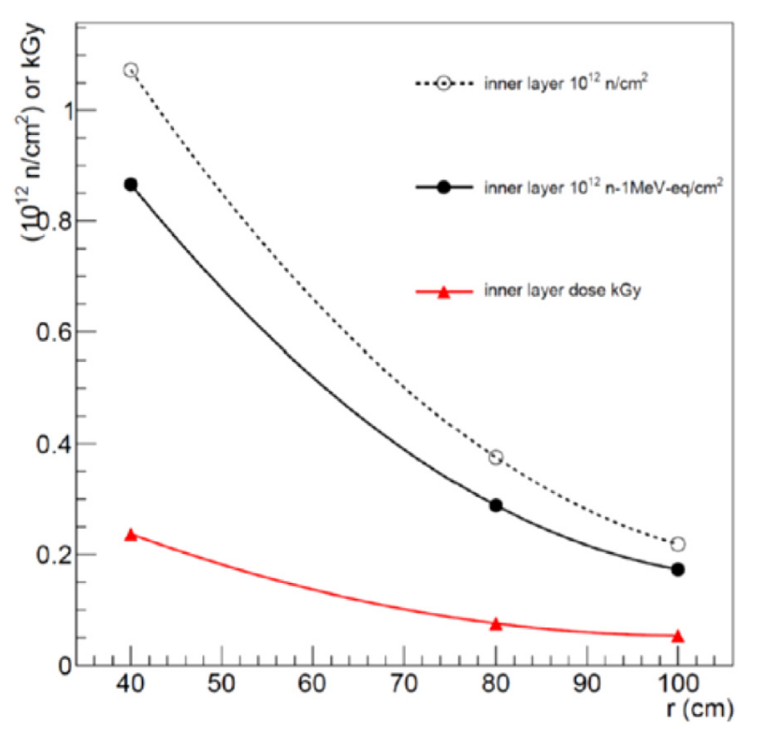

Fig. 4. Ionizing doses (red line, kGy) and non-ionizing fluences (black dashed line: $\mathrm{n} / \mathrm{cm}^{2}$; black continuous line: $1 \mathrm{MeV}$-equivalent $\mathrm{n} / \mathrm{cm}^{2}$ ) as a function of the distance between the axis of the ENUBET decay tunnel and the inner radius of the calorimeter.

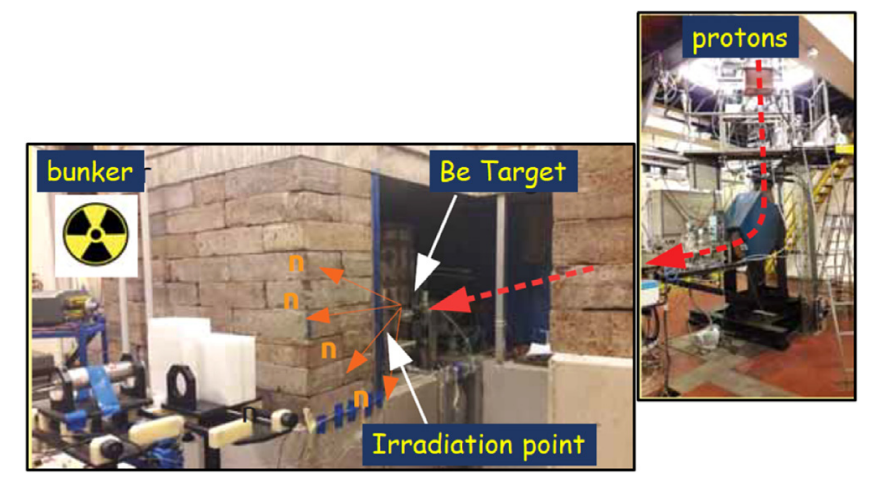

Fig. 5. Picture of the LNL infrastructure used for irradiation tests.

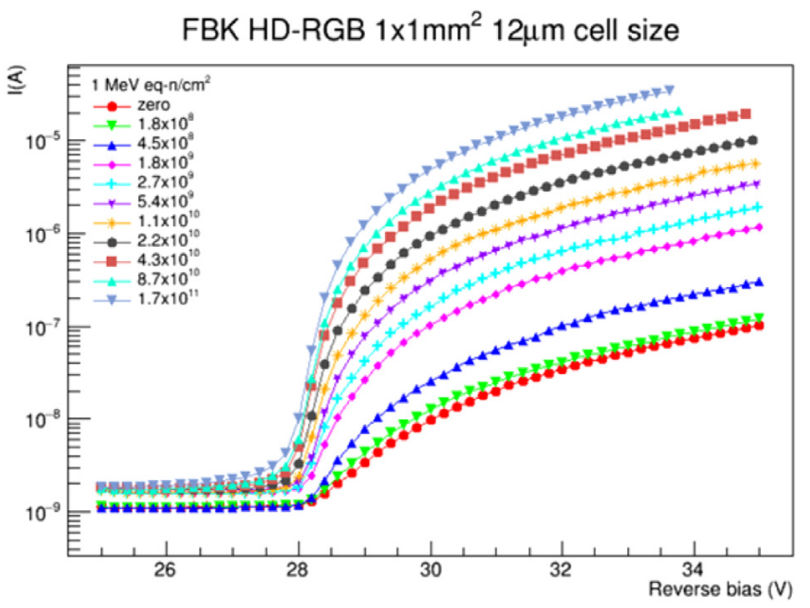

Fig. 6. I-V curve of the single-SiPM PCB at different irradiation levels expressed in $\mathrm{n} / \mathrm{cm}^{2}$.

1024 oscilloscope to record the waveforms. Between two irradiation sessions the current as a function of the overvoltage was measured for all the PCB boards and in Fig. 6 the IV measurements are shown as a function of the integrated dose for the single $12 \mu \mathrm{m}$ SiPM test board.

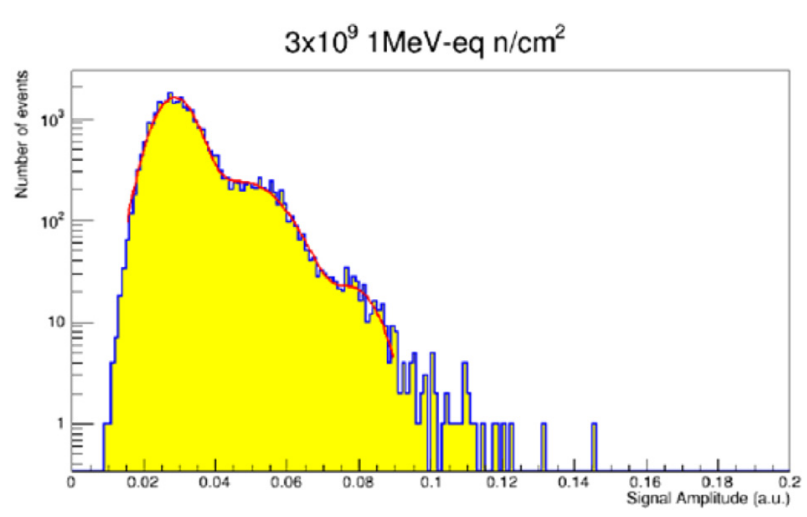

Fig. 7. Signal peak distribution at $3 \times 10^{9} \mathrm{n} / \mathrm{cm}^{2}$ for the single-SiPM PCB $(12 \mu \mathrm{m}$ cell-pitch, $1 \mathrm{~mm}^{2} \mathrm{SiPM}$ ). Red line is the fit of the first three photoelectron peaks: for fluence greater than this the multi-peak structure is not visible anymore.

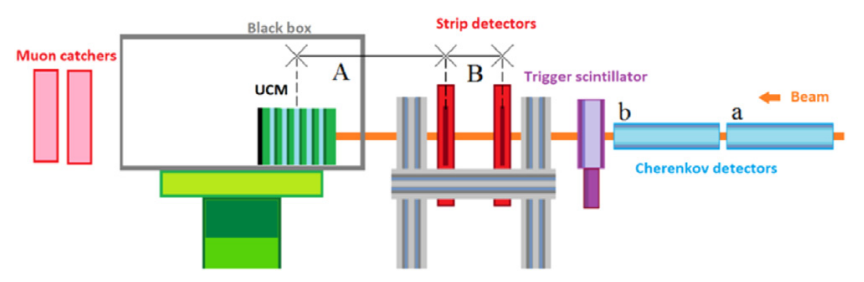

Fig. 8. Sketch of the experimental setup at the CERN T9 beamline.

Only minor changes are visible in the breakdown voltage with an increase by more than two orders of magnitude in dark current. Fig. 7 shows that the single photoelectron sensitivity is lost for fluences greater than $3 \times 10^{9} \mathrm{n} / \mathrm{cm}^{2}$.

After irradiation the samples were stored at $25 \pm 1{ }^{\circ} \mathrm{C}$ for about three months before installing the boards on the UCM at CERN. The behavior of the dark current versus time during the storage period has not being studied; however, before installation at CERN, the I-V curves showed a current reduction comparable to what expected by room temperature self-annealing reported in [7].

\section{Test beam}

The irradiated PCBs were tested in October 2017 at the T9 beamline of the CERN East Area facility. In Fig. 8 the setup used in the beam area is sketched. The particle beam is composed of electrons, muons and pions with momentum selectable between 1 and $5 \mathrm{GeV}$. The PCBs were tested with two different ENUBET UCMs:

- Prototype 16B. This is a standard UCM composed of EJ-200 scintillator tiles $\left(3 \times 3 \times 0.5 \mathrm{~cm}^{3}\right.$ each $)$ interspaced with iron slabs $\left(3 \times 3 \times 1.5 \mathrm{~cm}^{3}\right.$ each). The 9 WLS fibers are the Y11 produced by Kuraray. The number of photoelectrons due to minimum ionizing particles (MIP) crossing the whole UCM has been estimated by laboratory measurements with cosmic rays to be $\sim 50$.

- Prototype 17UA. This prototype was built from injection molded scintillator tiles produced by Uniplast and it consists of active area $\left(3 \times 3 \times 1.35 \mathrm{~cm}^{3}\right.$ each $)$ interleaved with iron absorber $(3 \times 3 \times 1.5$ $\mathrm{cm}^{3}$ each) equipped with the same WLS fibers used for the $16 \mathrm{~B}$ prototype. The number of photoelectrons due to MIP crossing the whole UCM has been estimated to be $\sim 85$.

These prototypes were tested using both a 9-SiPM board that was not irradiated at INFN-LNL and an irradiated board. The boards were equipped with $15 \mathrm{~m}$ cell-pitch SiPMs. By requiring a signal in both Cherenkov counters it is possible to select electrons, while muons and non-interacting pions correspond to events with no signal in the 


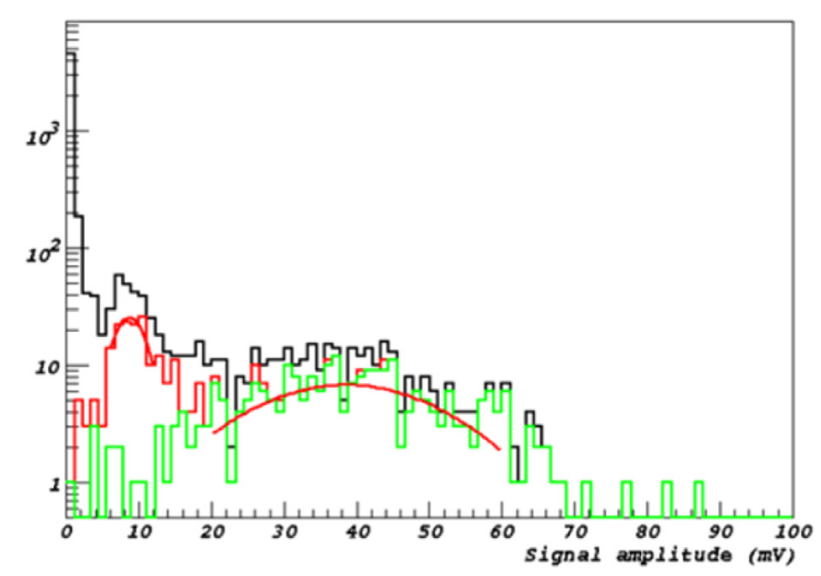

Fig. 9. UCM 16B equipped with a non-irradiated board. The black line shows all signals triggered during the run and it is dominated by dark counts, the red line is the MIP signal and the green one is electron signal. The red lines show the Gaussian fit of the signal peak for electrons and mip-like particles. (For interpretation of the references to color in this figure legend, the reader is referred to the web version of this article.)

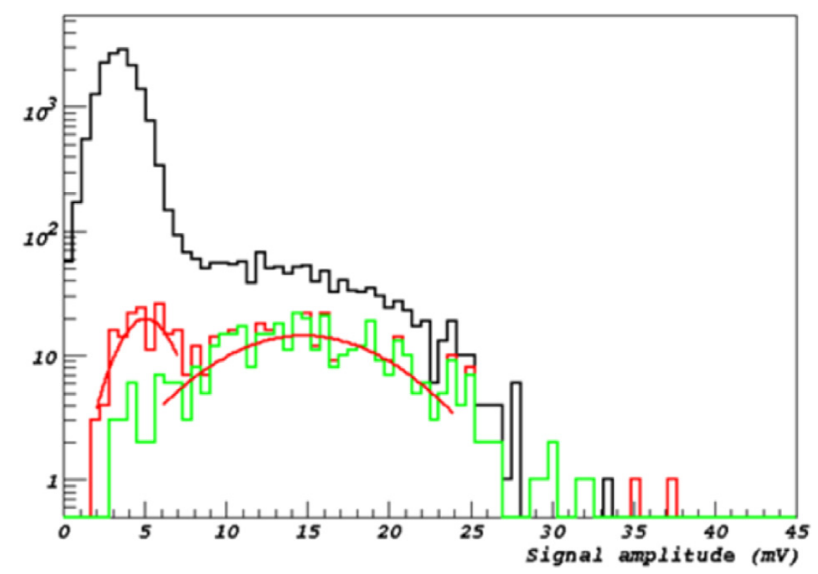

Fig. 10. UCM 16B equipped with a irradiated board: the black line shows all signals triggered during the run, red line is the MIP signal and green line is the electron signal. MIP signal is not separated by the background due to dark noise. The red lines show the Gaussian fit of the pedestal, signal peak for electrons and mip-like particles. (For interpretation of the references to color in this figure legend, the reader is referred to the web version of this article.)

Cherenkov counters and signal in a muon catcher. The silicon strip detectors are employed to select particles entering the front face of the UCM in a $2 \times 2 \mathrm{~cm}^{2}$ fiducial area and crossing the whole UCM. Fig. 9 shows the signal response of the UCM 16B equipped with the nonirradiated board to minimum ionizing particles (red line) and electrons (green line): MIP signal is well separated from the dark noise and the electrons are well separated from MIP signal. The red lines show the Gaussian fit of the signal peak for electrons and mip-like particles.

The results of Fig. 10 obtained with the irradiated board mounted on the same UCM 16B demonstrate that it is not possible to separate a MIP from the noise peak up to the maximum fluence expected in ENUBET $\left(2 \times 10^{11} \mathrm{n} / \mathrm{cm}^{2}\right)$ due to the increase of the dark counts, even if it has the capability to separate the electron peak from noise.

Preserving the capability to measure the MIP signal during the entire lifetime of the experiment is important because this signal can be used to monitor changes of the UCM response over time: it this way it is possible to equalize and compensate any amplitude losses by acting on the overvoltage.

The 17UA prototype has a larger scintillator thickness than the UCM $16 \mathrm{~B}$ and this is enough to preserve the separation of the MIP peak from

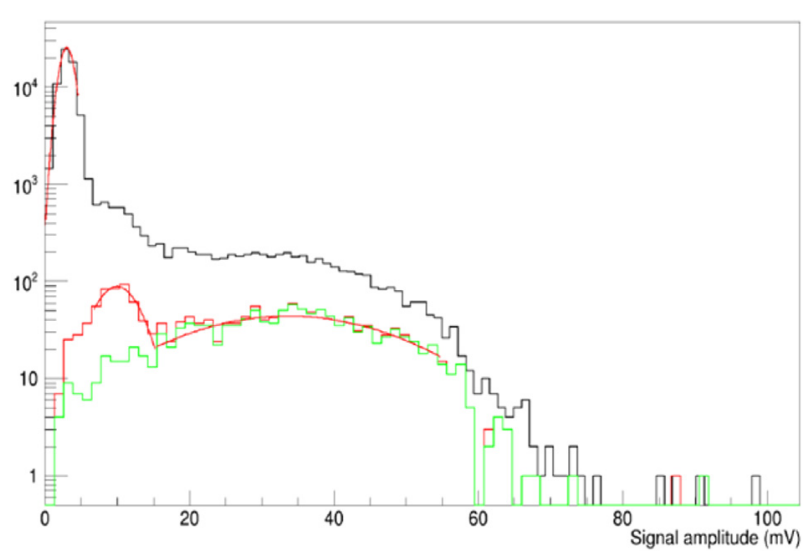

Fig. 11. UCM 17B equipped with an irradiated board. the black line show all signals triggered during the run, red line is the MIP signal and green line is electron signal. MIP signal is separated from the background due to dark noise. (For interpretation of the references to color in this figure legend, the reader is referred to the web version of this article.)
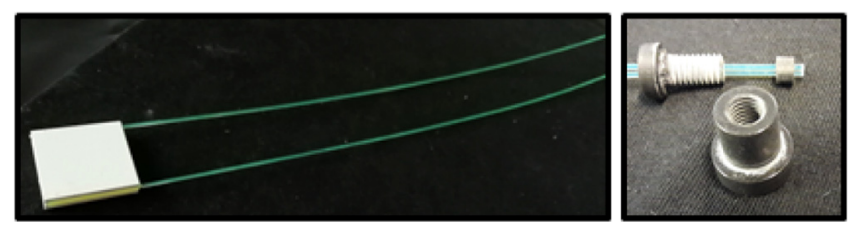

Fig. 12. Left: photon veto single tile EJ-204 scintillator with WLS fibers glued. Right: custom optical connector, to easily match SiPM with fibers.

the dark noise peak even after irradiation as it is shown in Fig. 11 for $1 \mathrm{GeV}$ particles.

Irradiation effects contribute to signal losses through a reduction of the gain $\times \mathrm{PDE}$ and of the transparency of the epoxy employed for the encapsulation of the SiPM. A signal reduction down to $\sim 50 \%$ can be recovered by increasing the bias voltage remaining well within the operation range of the FBK SiPM.

\section{Photon veto tests}

The photon veto, as discussed in Section 3, provides both photon identification capabilities and precise timing of the particles in the instrumented decay tunnel. The baseline performances (photon identification at $99 \%$ and time resolutions $<10 \mathrm{~ns}$ ) are strictly necessary to achieve the cross section measurement goals of ENUBET.

As it is shown in Fig. 12, each photon veto layer tested at T9 beamline of the CERN East Area facility consists of:

- one scintillator tile EJ-204 $3 \times 3 \times 0.5 \mathrm{~cm}^{3}$ produced by Eljen Technology;

- two WLS fibers $40 \mathrm{~cm}$ long, produced by Saint-Gobain and glued to the sides of the scintillator with an optical cement;

- a custom screw-optical connector which allows placement reproducibility of the fibers to a SiPM SenSL-30020J.

This setup provides a good uniformity and high light collection efficiency, greater then $99 \%$. In Fig. 13 a preliminary result concerning the separation of 1-2 particle as seen by a doublet.

The time resolution for this detector has been measured to be less then 500 ps, well within the requirement of the ENUBET project. Moreover the WLS fibers allow to keep the SiPM away from the bulk reducing the accumulated dose. A preliminary FLUKA simulation shows that, by putting in front of the SiPM $30 \mathrm{~cm}$ of borated polyethylene, it is possible to achieve a reduction factor of $\sim 18$ on the neutron fluence averaging over the spectrum as shown in Fig. 14. 


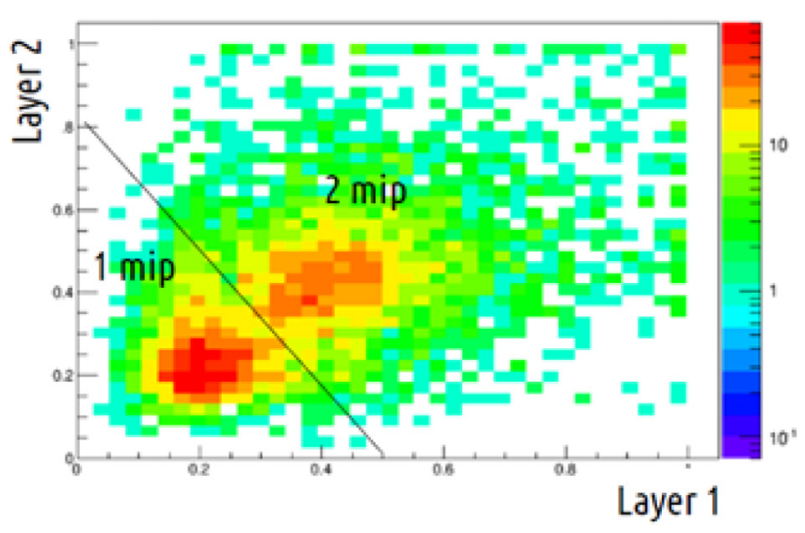

Fig. 13. Photon Veto doublet response to one and two particles.

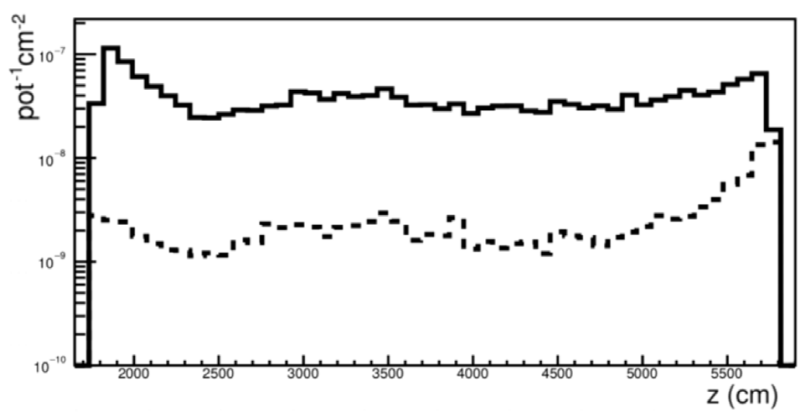

Fig. 14. Neutron fluence reduction estimated with a FLUKA simulation by putting $30 \mathrm{~cm}$ of borated polyethylene in front of SiPMs. The solid line represents the neutron flux at the inner surface of the tagger while the dashed one the flux just outside of the shielding showing a reduction of a factor $\sim 18$.

This alternative light readout strategy has been taken into account also for the calorimeter: a group of ten WLS fibers, corresponding to one UCM, $40 \mathrm{~cm}$ long are bundled to a single SiPM of $4 \times 4 \mathrm{~mm}^{2} \mathrm{SiPM}$ from Advansid.

\section{Conclusions}

The precision of neutrino cross section is a key point to establish $\mathrm{CP}$ violation in the leptonic sector for the next generation neutrino experiment and is currently dominated by the knowledge of the flux at source in conventional neutrino beams. A novel approach to the $v_{e}$ cross section measurement is based on the monitoring of large angle positrons coming from the three-body semileptonic decays of kaons.
In the baseline design the decay tunnel will be instrumented with a positron tagger (a shashlik calorimeter readout with SiPM) and a photon veto layer to suppress the $\pi_{0}$ background and provide timing informations. The SiPM used to instrument the calorimeter prototypes are produced by FBK company. In order to test the functionality of the sensors over the entire lifetime of the experiment, SiPM with different cell size have been irradiated at the INFN-LNL Irradiation Test facility and then tested with beam particle at CERN. It has been shown that the calorimeter is capable to operate up to a fluence of $2 \times 10^{11} \mathrm{n} / \mathrm{cm}^{2}$ providing that the scintillator thickness is increased form 0.5 up to $1.5 \mathrm{~cm}$ to preserve MIP identification. Photon veto prototypes are equipped with SiPM produced by SenSL company: preliminary tests conducted at beamline at CERN shows that it is possible to match the ENUBET requirements both in terms of time resolution and 1 particle/2 particle identification capability. The design of photon veto layer can be used to instrument also the calorimeter detector with the extra benefit of reducing by a factor $\sim 18$ the fluence on SiPMs.

\section{Declaration of competing interest}

The authors declare that they have no known competing financial interests or personal relationships that could have appeared to influence the work reported in this paper.

\section{Acknowledgments}

This project has received funding from the European Research Council (ERC) under the European Union's Horizon 2020 research and innovation programme (G.A. n. 681647) and by the Italian Ministry of Research (MIUR) within the "Progetto FARE" 2016, NUTECH, "NeUtrino Time-tagged bEams with CHerenkov detectors", R1623F4S38).

\section{References}

[1] A. Longhin, L. Ludovici, F. Terranova, A novel technique for the measurement of the electron neutrino cross section, Eur. Phys. J. C75 (2015) 155, http: //dx.doi.org/10.1140/epjc/s10052-015-3378-9.

[2] A. Berra, et al., Enabling precise measurements of flux in accelerator neutrino beams: the enubet project, 2014, CERN-SPSC-2016-036, SPSC-EOI-014.

[3] A. Berra, et al., A compact light readout system for longitudinally segmented shashlik calorimeters, Nucl. Instrum. Methods A830 (2016) 345-354, http://dx. doi.org/10.1016/j.nima.2016.05.123.

[4] A. Berra, et al., Shashlik calorimeters with embedded sipms for longitudinal segmentation, IEEE Trans. Nucl. Sci. 64 (2017) 1056-1061, http://dx.doi.org/10. 1109/TNS.2017.2672500.

[5] G. Ballerini, et al., Testbeam performance of a shashlik calorimeter with finegrained longitudinal segmentation, JINST 13 (2018) P01028, http://dx.doi.org/ 10.1088/1748-0221/13/01/P01028.

[6] F. Acerbi, et al., Irradiation and performance of rgb-hd silicon photomultipliers for calorimetric applications, JINST 14 (2019) P02029, http://dx.doi.org/10.1088/ 1748-0221/14/02/P02029.

[7] Y. Qiang, C. Zorn, F. Barbosa, E. Smith, Radiation hardness tests of SiPMs for the JLab Hall D barrel calorimeter, Nucl. Instrum. Methods A698 (2013) 234-241, http://dx.doi.org/10.1016/j.nima.2012.10.015. 